\title{
Galaxy Metabolism
}

\author{
Andrew Hopkins ${ }^{\mathrm{A}}$ \\ A Anglo-Australian Observatory, P.O. Box 296, Epping, NSW 1710, Australia
}

'Galaxy Metabolism' was the second in the annual 'Southern Cross Astrophysics Conference Series' (http: // www .aao.gov.au/AAO/southerncross/), supported by the Anglo-Australian Observatory and the Australia Telescope National Facility. It was held at the Australian National Maritime Museum in Darling Harbour, Sydney, from 22 to 26 June 2009, and was attended by 91 delegates from around the world.

Over the past decade, both the star formation history and stellar mass density in galaxies spanning most of cosmic history have been well constrained. This provides the backdrop and framework within which many detailed investigations of galaxy growth are now placed. The mass-dependent and environment-dependent evolution of galaxies over cosmic history is now the focus of several surveys. Many studies are also exploring the role of gas infall and outflow in driving galaxy evolution, and the connection of these processes to massive star formation within galaxies.

The aims of 'Galaxy Metabolism' were to bring together the global constraints on galaxy evolution, at both low and high redshift, with detailed studies of well-resolved systems, to define a clear picture of our understanding of galaxy metabolism: How do the processes of ingestion (infall), digestion (ISM physics, star formation) and excretion (outflow) govern the global properties of galaxies; how do these change over a galaxy's lifetime; and are the constraints from nearby well resolved studies consistent with those from large population surveys at low and high redshift?

The conference was a great success, with an extensive variety of topics covered spanning many aspects of galaxy evolution, and brought together eloquently in a comprehensive conference summary by Warrick Couch. The four papers by De Lucia (2010), Cole (2010), Vlajić (2010) and Stocke et al. (2010) presented in this special collection of PASA are just a sampling of the depth and variety of the presentations given during the conference.

\section{References}

Cole, A., 2010, PASA, 27, 234

De Lucia, G., 2010, PASA, 27, 242

Stocke, J. T., Keeney, B. A. \& Danforth, C. W., 2010, PASA, 27, 256 Vlajić, M., 2010, PASA, 27, 252 